\title{
A Systematic CCSD(T) Study of Long-Range and Noncovalent Interactions between Benzene and a Series of First- and Second-Row Hydrides and Rare Gas Atoms
}

\author{
Deborah L. Crittenden* \\ Research School of Chemistry, Australian National University, ACT, Australia 0200
}

Received: October 15, 2008; Revised Manuscript Received: December 17, 2008

\begin{abstract}
Binding energies, potential energy curves, and equilibrium intermonomer distances describing the interaction between benzene and a series of first- and second-row hydrides and rare gas atoms are calculated using coupled-cluster theory with single, double, and perturbative triple excitations $(\operatorname{CCSD}(\mathrm{T}))$ in conjunction with a large augmented quadruple- $\zeta$ basis set (aug-cc-pVQZ). These benchmark results are accurate to within one eighth of $1 \mathrm{kcal} / \mathrm{mol}$ and, as such, provide a reliable foundation for the development and testing of more approximate methods for calculating long-range and noncovalent interactions.
\end{abstract}

\section{Introduction}

Long-range and noncovalent interactions play a key role in a large range of physicochemical processes, from driving the condensation of inert gases through determining the phases and properties of liquid crystals to influencing the structure and folding of proteins and nucleic acids. Although approximate methods for describing these interactions have long been utilized in molecular mechanics and molecular dynamics simulations, ${ }^{1}$ it is only recently that the development of more accurate models has become the focus of the density functional theory (DFT) community. ${ }^{2-9}$ However, the development of accurate models requires high-quality benchmark data for calibration and comparison, and a number of authors have noted that very few high-quality potential energy curves are available for prototype noncovalent interactions. ${ }^{2,10}$ In particular, Grimme has recently noted that very accurate $\mathrm{ab}$ initio data are still missing for complexes of benzene with small molecules. ${ }^{2}$ Therefore, the purpose of this paper is to build on previous studies of benzene-small molecule complexes ${ }^{10-26}$ to provide equilibrium binding energies $\left(D_{\mathrm{e}}\right)$ and potential energy curves with "subchemical" accuracy (i.e., accurate to within a fraction of a $\mathrm{kcal} / \mathrm{mol}$ ) for such systems. For systems where the zero-point energy has also been accurately determined, the vibrationally averaged binding energy $\left(D_{0}\right)$ is calculated and compared with experimental results. ${ }^{27-45}$ For clarity, the results of all previous experimental and high-level ab initio studies on benzene-small molecule systems are summarized in Table 1 .

\section{Methods Section}

Initial conformations of all benzene-small molecule complexes are illustrated in Figure 1. Within these complexes, each monomer was held rigid over all intermolecular distances, and monomer geometries were assigned using the best parameters currently available in the literature. For reference, geometric parameters for all monomers (benzene, $\mathrm{Ne}, \mathrm{HF}, \mathrm{H}_{2} \mathrm{O}, \mathrm{NH}_{3}, \mathrm{CH}_{4}, \mathrm{Ar}, \mathrm{HCl}, \mathrm{H}_{2} \mathrm{~S}, \mathrm{PH}_{3}, \mathrm{SiH}_{4}$ ) and their corresponding literature references are summarized in Table 2. ${ }^{46-54}$ Initial intermolecular distances and orientations were assigned according to previous ab initio and experimental

\footnotetext{
*E-mail: deborah@rsc.anu.edu.au.
}

studies of benzene-small molecule complexes. ${ }^{10-45}$ It has long been known that neon and argon form complexes with $C_{6 v}$ symmetry, ${ }^{11,12,25,27,28,38-40}$ as illustrated in Figure 1. It is also well-established that ammonia-benzene complexes possess $C_{2 v}$ symmetry, with a single proton pointing directly toward the center of the benzene ring. ${ }^{35,36}$ Experiment and theory also agree that the minimum-energy structure of the methane-benzene complex is $C_{3 v}$-symmetric with a single $\mathrm{C}-\mathrm{H}$ bond pointing toward the benzene ring, ${ }^{21-24,37}$ and the $\mathrm{HCl}$-benzene complex was assigned as a $C_{6 v}$-symmetric structure by consensus between experimental and theoretical results. ${ }^{26,42-44}$ Assigning the orientations of hydrogen fluoride, water, and dihydrogen sulfide relative to the benzene ring was more contentious, as the vibrationally averaged structures observed in experimental studies differ significantly from the equilibrium structures obtained ab initio. ${ }^{10,13-20,29-34,45}$ In all cases, however, the vibrationally averaged structures are more symmetric than their equilibrium counterparts and, therefore, provide a more convenient starting point for subsequent potential energy curve (PEC) mapping. The vibrationally averaged structure of the hydrogen fluoride complex possesses $C_{6 v}$ symmetry, with the electropositive proton of $\mathrm{HF}$ pointing toward the center of the benzene ring. $\mathrm{H}_{2} \mathrm{O}$ and $\mathrm{H}_{2} \mathrm{~S}$ both adopt a $C_{2 v}$-symmetric orientation, with their heteroatoms directly above the center of the benzene ring and both protons pointing down toward the ring. Finally, in the absence of any previous studies on complexes of $\mathrm{PH}_{3}$ and $\mathrm{SiH}_{4}$ with benzene, intermolecular orientations of these complexes were assigned by analogy with their first-row counterparts.

Potential energy curves (PECs) along the intermonomer distance $\left(R^{\mathrm{cm}-\mathrm{X}}\right)$ were obtained using second-order Moller-Plesset perturbation theory (MP2) and coupled-cluster theory including single, double, and perturbative triple substitutions $(\operatorname{CCSD}(\mathrm{T}))^{55}$ in conjunction with the correlation-consistent basis settings developed by Dunning and co-workers. ${ }^{56,57}$ MP2 energies were determined using augmented triple- and quadruple- $\zeta$ basis sets (aug-cc-pVTZ and aug-cc-pVQZ), while $\operatorname{CCSD}(\mathrm{T})$ energies were obtained using only the aug-cc-pVTZ basis. CCSD(T)/ aug-cc-pVQZ energies were then estimated using the following formulas 


$$
\begin{gathered}
\text { est. } E^{\mathrm{CCST}(\mathrm{T}) \text { /aug-cc-pVQZ }}=E^{\mathrm{MP} 2 \text { aug-cc-pVQZ }}+\Delta_{\mathrm{MP} 2}^{\mathrm{CCSD}(\mathrm{T})} \\
\Delta_{\mathrm{MP} 2}^{\mathrm{CCSD}(\mathrm{T})}=E^{\mathrm{CCSD}(\mathrm{T}) \text { /aug-cc-pVTZ }}-E^{\mathrm{MP} 2 \text { /aug-cc-pVTZ }}
\end{gathered}
$$

Basis set superposition error in the MP2/aug-cc-pVQZ energies was accounted for by using the counterpoise (CP) correction method of Boys and Bernardi ${ }^{58,59}$

$$
E_{\mathrm{AB}}^{\mathrm{BSSE}-\text { free }}=E_{\mathrm{AB}}^{\mathrm{AB}}-E_{\mathrm{A}}^{\mathrm{AB}}-E_{\mathrm{B}}^{\mathrm{AB}}+E_{\mathrm{A}}^{\mathrm{A}}+E_{\mathrm{B}}^{\mathrm{B}}
$$

where A and B denote fragments in a complex, the subscripted index signifies the system under consideration, and the superscripted index specifies the basis functions to be included in the calculation, irrespective of whether or not the corresponding nuclei are present. BSSE in the high-level correction term $\left(\Delta_{\mathrm{MP2}}^{\mathrm{CCS}(\mathrm{T})}\right)$ was assumed to be zero due to cancellation of errors between the $\operatorname{CCSD}(\mathrm{T})$ and MP2 energies. This was checked for a handful of cases and found to be a very accurate approximation.

TABLE 1: Summary of All Previous Experimental and High-Level Ab Initio Studies on Benzene-Small Molecule Complexes

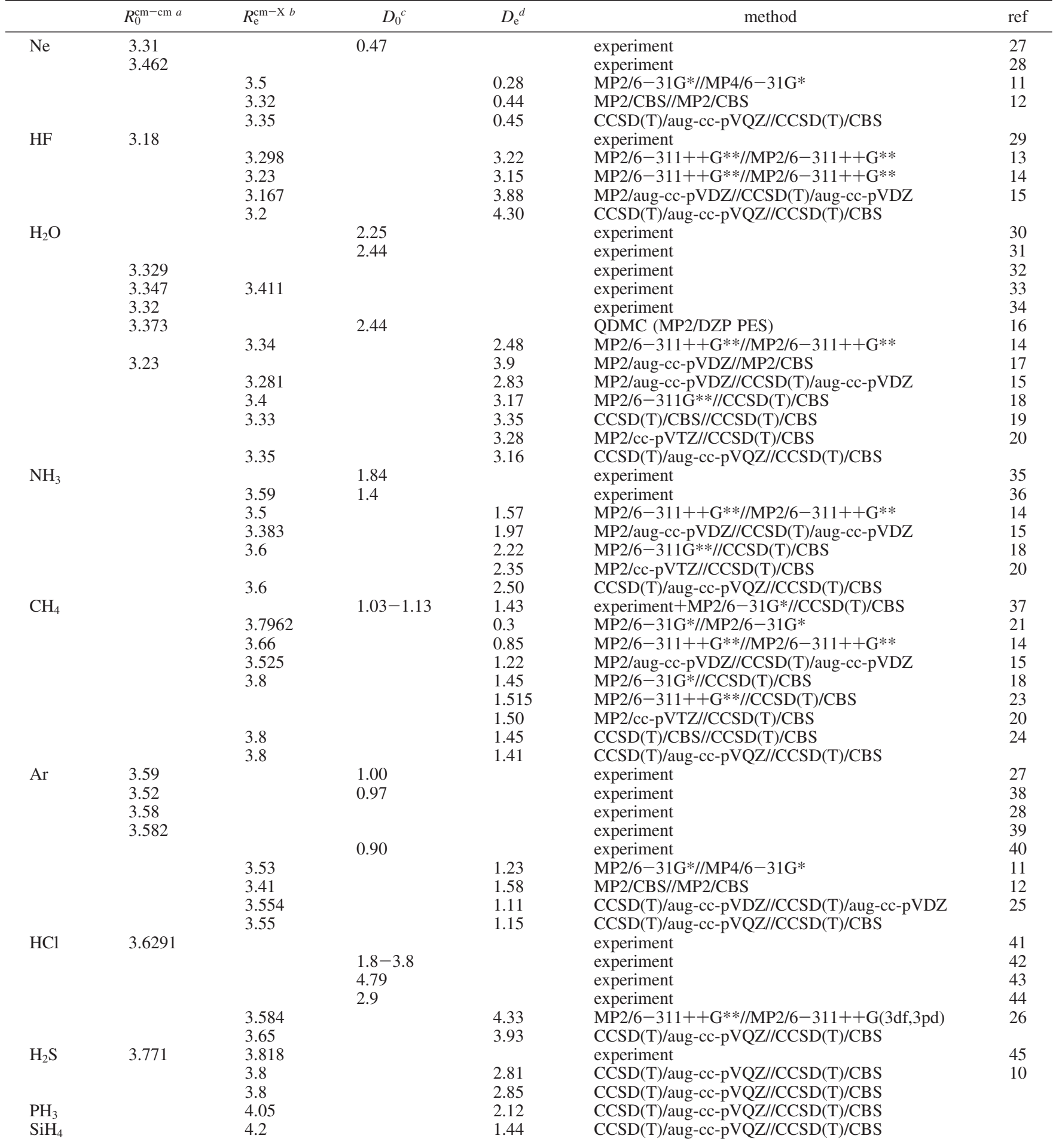

${ }^{a} R_{0}^{\mathrm{cm}-\mathrm{cm}}$ : the vibrationally averaged distance between the center of mass of benzene and the center of mass of the associated hydride. ${ }^{b} R_{\mathrm{e}}^{\mathrm{cm}-\mathrm{X}}$ : the equilibrium distance between the center of mass of benzene and the heteroatom of the associated hydride. ${ }^{c} D_{0}$ : the vibrationally averaged binding energy. ${ }^{d} D_{\mathrm{e}}$ : the equilibrium binding energy. 
TABLE 2: Best-Available Literature Values of Geometric Parameters for Benzene and a Series of First- and Second-Row Hydrides

\begin{tabular}{llc}
\hline system & geometry (angstrom and degrees) & ref \\
\hline benzene & $r_{\mathrm{CC}}=1.3915, r_{\mathrm{CH}}=1.0800$ & 46 \\
$\mathrm{HF}$ & $r_{\mathrm{HF}}=0.91696$ & 47 \\
$\mathrm{H}_{2} \mathrm{O}$ & $r_{\mathrm{OH}}=0.95785, \theta_{\mathrm{HOH}}=104.501$ & 48 \\
$\mathrm{NH}_{3}$ & $r_{\mathrm{NH}}=1.0110, \theta_{\mathrm{HNH}}=106.70$ & 49 \\
$\mathrm{CH}_{4}$ & $r_{\mathrm{CH}}=1.0110$ & 50 \\
$\mathrm{HCl}$ & $r_{\mathrm{HCl}}=1.2747$ & 51 \\
$\mathrm{H}_{2} \mathrm{~S}$ & $r_{\mathrm{SH}}=1.3356, \theta_{\mathrm{HSH}}=92.12$ & 52 \\
$\mathrm{PH}_{3}$ & $r_{\mathrm{PH}}=1.41105, \theta_{\mathrm{HPH}}=93.4970$ & 53 \\
$\mathrm{SiH}_{4}$ & $r_{\mathrm{SiH}}=1.4734$ & 54
\end{tabular}

Finally, Hartree-Fock, MP2, and CCSD(T) interaction energies at the complete basis set (CBS) limit were estimated using the two-point extrapolation formula of Helgaker et al. ${ }^{60}$

$$
E_{\mathrm{CBS}} \approx \frac{X^{3} E(X)-(X-1)^{3} E(X-1)}{X^{3}-(X-1)^{3}} \quad X=4
$$

Although this approach differs from the conventional procedure for extrapolation of Hartree-Fock energies, the HF/aug-ccpVQZ interaction energies are so close to the basis set limit that the extrapolation procedure is effectively immaterial. Therefore, this procedure is carried out only for consistency in the Hartree-Fock case.

Intermonomer distances were set to their $\operatorname{CCSD}(\mathrm{T}) /$ aug-ccpVQZ optimized values (determined to within $0.05 \AA$ ), while PEC scans over all torsional degrees of freedom were carried out at MP2/aug-cc-pVTZ. The torsional degrees of freedom explored in this study are illustrated in Figure 1. As these rotational modes have high symmetry and low complexity, it is reasonable to expect that a MP2/aug-cc-pVTZ scan using $10^{\circ}$ increments will be capable of accurately identifying all local and global minima, and a higher-level investigation of these torsional degrees of freedom is unwarranted. Finally, equilibrium structures were defined according to the est. CCSD(T)/aug-ccpVQZ optimized distances and MP2/aug-cc-pVTZ optimized torsional parameters (where applicable) for each complex. $\operatorname{CCSD}(\mathrm{T}) / \mathrm{CBS}$ equilibrium binding energies were calculated, within the rigid monomer approximation, using the $\operatorname{CCSD}(\mathrm{T}) /$ aug-cc-pVQZ approximation and CBS extrapolation schemes described above.

Reduced variational space self-consistent field (RVS-SCF) analysis, as implemented in the GAMESS suite of ab initio quantum chemical software, ${ }^{61,62}$ was carried out at each equilibrium geometry to partition the Hartree-Fock interaction energies into physically meaningful components. To complete the energy decomposition analysis, dispersion energies were added separately as the difference between CCSD(T)/CBS and $\mathrm{HF} / \mathrm{CBS}$ results.

All coupled-cluster and MP2 calculations were carried out using the MOLPRO suite of quantum chemical programs. ${ }^{63}$

\section{Results and Discussion}

Binding Energies. Equilibrium binding energies were calculated using a hierarchy of ab initio methods (Hartree-Fock, MP2, CCSD, CCSD(T)) and with a range of correlationconsistent basis sets (aug-cc-pVTZ, aug-cc-pVQZ, extrapolation to CBS limit). CBS limit energies for all ab initio methods are presented in Table 3, and $\operatorname{CCSD}(\mathrm{T})$ energies obtained using a range of different basis sets are presented in Table 4. Estimates of the deviation of calculated CCSD(T)/CBS binding energies from exact are given in Table 5. Full details of all intermolecular interaction potentials may be obtained as Supporting Information.

From Table 3, we observe that the results obtained using Hartree-Fock theory are both quantitatively and qualitatively incorrect. In particular, Hartree-Fock theory predicts that the majority of benzene-small molecule complexes considered here are unbound at their CCSD(T)/aug-cc-pVQZ optimized geometries. The only exceptions to this are complexes that include $\mathrm{HF}, \mathrm{HCl}$, or $\mathrm{H}_{2} \mathrm{O}$. This implies that dispersion interactions, which are neglected by Hartree-Fock theory, are the main attractive force stabilizing weakly bound molecular complexes, except in the case of systems whose binding behavior is dominated by relatively strong electrostatic interactions.

The data presented in Table 3 also show that both MP2 and CCSD do a reasonable job of recovering the dispersion-based component of the correlation energy. This is more clearly illustrated in Figure 2, where the CBS limit binding energies obtained using MP2, spin-component-scaled MP2 (SCS-MP2), ${ }^{64}$ and CCSD are plotted against the "exact" CCSD(T)/CBS energies. As observed previously, MP2 has a tendency to overbind, while CCSD has a tendency to underbind, relative to the $\operatorname{CCSD}(\mathrm{T})$ reference. ${ }^{10,24}$ It is surprising, though, that the magnitude of the error in the MP2 and CCSD correlation energies is approximately equal. It is also surprising that SCSMP2 performs significantly better than either MP2 or CCSD, suggesting that this method provides an affordable route to calculating accurate interaction energies for predominantly dispersion-bound systems. This is in agreement with recent results obtained by Bachorz et al. ${ }^{65}$ and Takatani et al.,${ }^{66}$ who also found that SCS-MP2 dissociation curves of dispersionbound complexes closely follow their $\operatorname{CCSD}(\mathrm{T})$ counterparts. However, Bachorz et al. ${ }^{65}$ warn that SCS-MP2 underestimates the binding energy of hydrogen-bonded complexes and thus cannot attain $\operatorname{CCSD}(\mathrm{T})$ quality for systems where dispersion and hydrogen bonding are competitive.

Systematic error analysis begins by assuming that the perturbative triples contribution to the binding energy (given by the difference between the CCSD/CBS and CCSD(T)/CBS results) provides an upper bound to the post-triples contributions that have been neglected in the present work. This assumption yields a maximum relative error of $35 \%$ for $\mathrm{SiH}_{4}$ and a minimum relative error of $10 \%$ for HF. At face value, these large relative errors suggest that the $\operatorname{CCSD}(\mathrm{T})$ method is far from convergence in its treatment of long-range electron correlation effects. However, recent work by Hopkins and Tschumper ${ }^{67}$ has shown that quadruples contributions to the binding energy are typically an order of magnitude smaller than triples contributions for van der Waals dimers, and higher excitations are expected to contribute even less. Therefore, in the present work, a more realistic estimate of the post-triples contribution to the binding energy is obtained by dividing the triples contribution by a factor of 10 . This reduces the maximum relative error across all systems to less than $3.5 \%\left(\mathrm{SiH}_{4}\right)$ and the maximum absolute error to $0.07 \mathrm{kcal} / \mathrm{mol}\left(\mathrm{H}_{2} \mathrm{~S}\right)$. However, it is also necessary to take into account the effect of treating the triples contributions perturbatively, as opposed to using the full iterative triples method. Previous work by Pittner and Hobza ${ }^{68}$ has shown that this effect is small (less than $1 \%$ for the system most closely related to those in the present work) and that the CCSDT energies show a slight destabilization relative to the $\operatorname{CCSD}(\mathrm{T})$ results. As this effect is smaller than the post-triples contributions and opposite in sign, the post-triples errors still provide an upper bound to the total correlation treatment incompleteness error. 


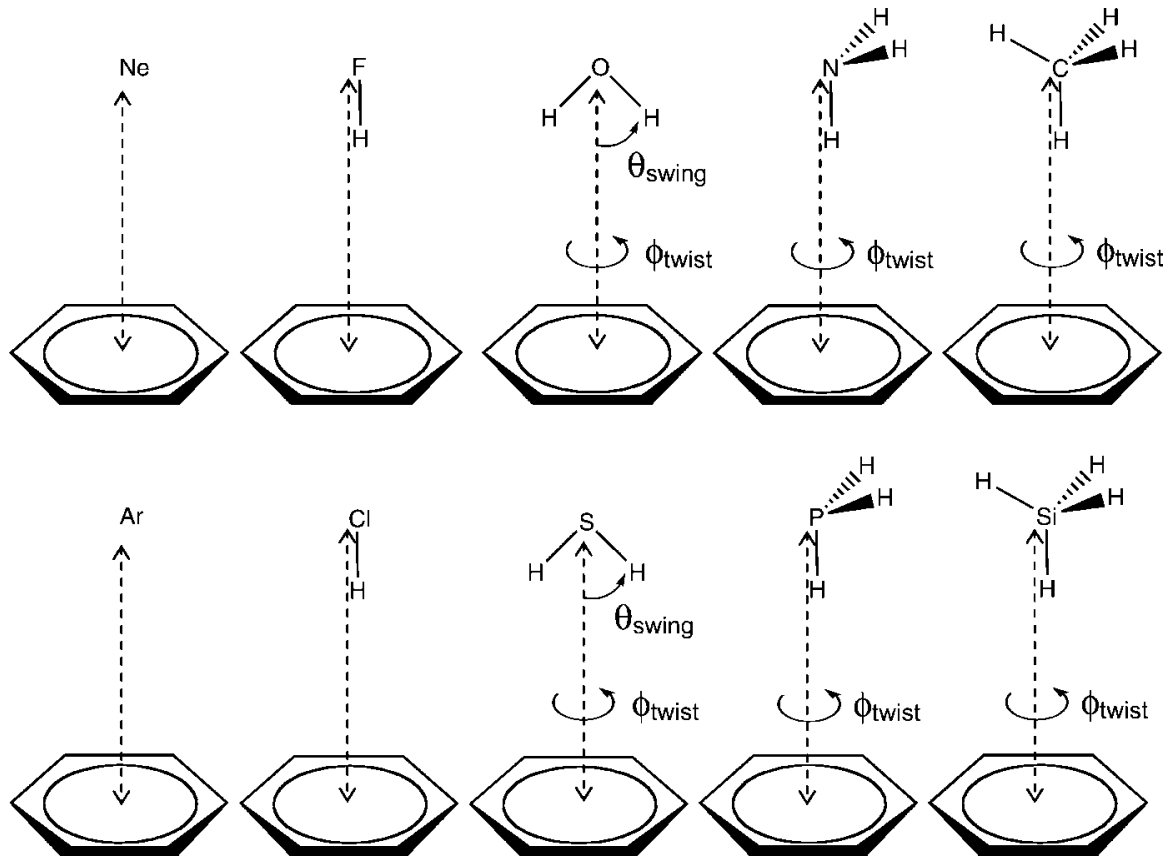

Figure 1. Initial conformations of all benzene-small molecule complexes.

TABLE 3: CBS Limit Equilibrium Interaction Energies $\left(D_{\mathrm{e}}, \mathrm{kcal} / \mathrm{mol}\right)$

\begin{tabular}{lcccccrrrrr}
\hline & $\mathrm{Ne}$ & $\mathrm{HF}$ & $\mathrm{H}_{2} \mathrm{O}$ & \multicolumn{1}{c}{$\mathrm{NH}_{3}$} & $\mathrm{CH}_{4}$ & $\mathrm{Ar}$ & $\mathrm{HCl}$ & $\mathrm{H}_{2} \mathrm{~S}$ & $\mathrm{PH}_{3}$ & $\mathrm{SiH}_{4}$ \\
\hline Hartree-Fock & 0.35 & -2.20 & -0.63 & 0.13 & 0.87 & 0.89 & -0.63 & 0.71 & 1.13 & 1.39 \\
MP2 & -0.44 & -4.50 & -3.45 & -2.90 & -1.79 & -1.58 & -4.85 & -3.74 & -2.87 & -1.87 \\
SCS-MP2 & -0.27 & -3.89 & -2.76 & -2.25 & -1.20 & -1.03 & -3.85 & -2.73 & -1.98 & -1.16 \\
CCSD & -0.34 & -3.88 & -2.66 & -2.03 & -0.99 & -0.82 & -3.29 & -2.16 & -1.49 & -0.94 \\
CCSD(T) & -0.45 & -4.30 & -3.16 & -2.50 & -1.41 & -1.15 & -3.93 & -2.85 & -2.12 & -1.44
\end{tabular}

TABLE 4: Convergence of $\operatorname{CCSD}(\mathrm{T})$ Interaction Energies $\left(D_{\mathrm{e}}, \mathrm{kcal} / \mathrm{mol}\right)$ toward the Complete Basis Set Limit

\begin{tabular}{lccccccccccc}
\hline & $\mathrm{Ne}$ & $\mathrm{HF}$ & $\mathrm{H}_{2} \mathrm{O}$ & $\mathrm{NH}_{3}$ & $\mathrm{CH}_{4}$ & $\mathrm{Ar}$ & $\mathrm{HCl}$ & $\mathrm{H}_{2} \mathrm{~S}^{2}$ & $\mathrm{PH}_{3}$ & $\mathrm{SiH}_{4}$ \\
\hline aug-cc-pVTZ & -0.37 & -4.06 & -2.99 & -2.43 & -1.37 & -1.02 & -3.70 & -2.72 & -2.04 & -1.40 \\
aug-cc-pVQZ & -0.42 & -4.20 & -3.09 & -2.47 & -1.39 & -1.10 & -3.83 & -2.79 & -2.08 & -1.42 \\
CBS & -0.45 & -4.30 & -3.16 & -2.50 & -1.41 & -1.15 & -3.93 & -2.85 & -2.12 & -1.44
\end{tabular}

TABLE 5: Estimated Deviation of Calculated CCSD(T)/CBS Binding Energies $\left(D_{\mathrm{e}}, \mathrm{kcal} / \mathrm{mol}\right)$ from Exact Due to Basis Set Extrapolation/Incompleteness Errors and Post-Triples Contributions

\begin{tabular}{lcccccccccc}
\hline & $\mathrm{Ne}$ & $\mathrm{HF}$ & $\mathrm{H}_{2} \mathrm{O}$ & $\mathrm{NH}_{3}$ & $\mathrm{CH}_{4}$ & $\mathrm{Ar}$ & $\mathrm{HCl}$ & $\mathrm{H}_{2} \mathrm{~S}$ & $\mathrm{PH}_{3}$ & $\mathrm{SiH}_{4}$ \\
\hline basis set incompleteness & \pm 0.02 & \pm 0.05 & \pm 0.03 & \pm 0.02 & \pm 0.01 & \pm 0.03 & \pm 0.05 & \pm 0.03 & \pm 0.02 & \pm 0.01 \\
post-triples contributions & -0.01 & -0.04 & -0.05 & -0.05 & -0.04 & -0.03 & -0.06 & -0.07 & -0.06 & -0.05 \\
maximum total error & -0.03 & -0.09 & -0.08 & -0.07 & -0.05 & -0.06 & -0.11 & -0.10 & -0.08 & -0.06 \\
max percentage error & 7 & 2 & 3 & 3 & 4 & 5 & 3 & 3 & 4 & 4
\end{tabular}

Therefore, final estimates of all correlation treatment incompleteness errors are encompassed by the post-triples contributions to the correlation energy provided in Table 5.

A second factor responsible for the difference between these results and exact binding energies is basis set incompleteness. From Table 4, we observe that the extrapolated CCSD(T)/CBS energies are converged to within $0.1 \mathrm{kcal} / \mathrm{mol}$, implying that the aug-cc-pVQZ basis is near completeness for these systems and basis set incompleteness errors are small. Another potential source of basis set incompleteness error arises from the basis set dependence of the $\Delta_{\mathrm{MP2}}^{\mathrm{CCSD}(\mathrm{T})}$ term (eq 2). However, evaluating this term using the aug-cc-pVDZ basis instead of the aug-ccpVTZ basis for the neon-benzene complex yields results that are almost identical (to within $0.006 \mathrm{kcal} / \mathrm{mol}$ ), indicating that the basis set dependence of this term is very weak and the basis set incompleteness error arising from it will be negligible. Therefore, basis set incompleteness errors are estimated only from the basis set extrapolation process as one-half of the

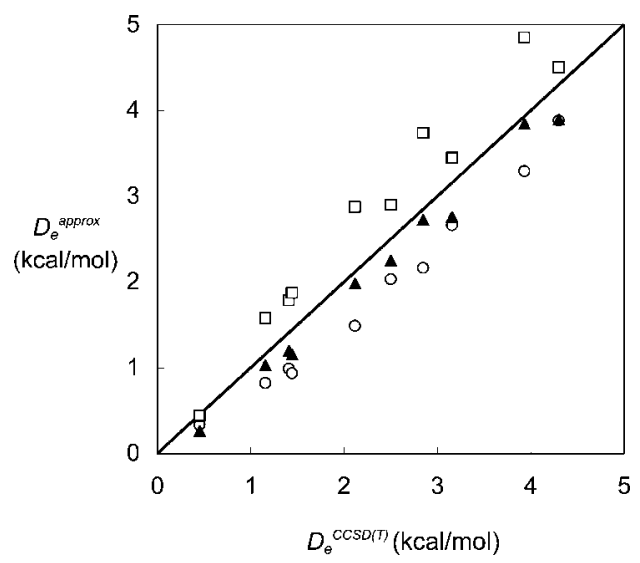

Figure 2. Comparison of near-exact $\operatorname{CCSD}(\mathrm{T}) / \mathrm{CBS}$ binding energies with CBS limit binding energies obtained using more approximate methods including MP2 (open square), SCS-MP2 (filled triangle), and CCSD (open circle). 
TABLE 6: RVS-SCF Decomposition of Interaction Energies $\left(D_{\mathrm{e}}, \mathrm{kcal} / \mathrm{mol}\right)^{a}$

\begin{tabular}{|c|c|c|c|c|c|c|c|c|c|c|}
\hline & $\mathrm{Ne}$ & $\mathrm{HF}$ & $\mathrm{H}_{2} \mathrm{O}$ & $\mathrm{NH}_{3}$ & $\mathrm{CH}_{4}$ & $\mathrm{Ar}$ & $\mathrm{HCl}$ & $\mathrm{H}_{2} \mathrm{~S}$ & $\mathrm{PH}_{3}$ & $\mathrm{SiH}_{4}$ \\
\hline electrostatic & -0.14 & -5.38 & -3.90 & -2.42 & -0.95 & -0.53 & -4.62 & -3.32 & -2.06 & -1.19 \\
\hline exchange & 0.49 & 3.18 & 3.27 & 2.29 & 1.82 & 1.43 & 3.99 & 4.03 & 3.19 & 2.58 \\
\hline dispersion & -0.80 & -2.10 & -2.53 & -2.37 & -2.28 & -2.05 & -3.30 & -3.56 & -3.25 & -2.83 \\
\hline
\end{tabular}

${ }^{a}$ The electrostatic component contains all multipole-multipole interactions (permanent and induced).

difference between CCSD(T)/aug-cc-pVQZ and CCSD(T)/CBS energies and are summarized in Table 5.

Total errors are estimated by simple addition of post-triples correlation energy corrections and basis set incompleteness errors. Maximum total and percentage errors are presented in Table 5. These data indicate that the binding energies calculated here deviate from the FCI/CBS limit by at most $7 \%$. Excluding the problem cases of neon and argon, where the binding energies are so small that a large relative error actually equates to a small absolute error, we find that the intrinsic accuracy of the CCSD(T)/CBS approach adopted here is, in fact, around $96 \%$, that is, $\operatorname{CCSD}(\mathrm{T}) / \mathrm{CBS}$ binding energies generally lie within $4 \%$ of the exact result.

The data presented in Table 3 can also be analyzed in terms of the likely physical origin of the attractive interaction in each benzene-small molecule complex. Trends in binding energies across each isoelectronic series are consistent with the behavior expected from simple electrostatic and dispersion models. For example, neon has a smaller binding energy than methane due to its smaller size and lower polarizability. Both complexes have lower binding energies than those that include polar molecules, where the binding energy increases monotonically with polarity. Trends in binding energies between isoelectronic series show an interesting balance between electrostatic and dispersion effects. For complexes involving nonpolar molecules, the more polarizable second-row systems have larger binding energies than their first-row counterparts, as expected from classical dispersion theory. However, for complexes with polar molecules, the situation is exactly reversed, and it is the first-row systems that have larger binding energies. This is consistent with classical electrostatic interaction theory, which predicts that the more polar first-row complexes should have stronger interactions than the less polar second-row complexes. This suggests that, where present, electrostatic interactions tend to dominate dispersion interactions in weakly bound complexes. This issue will be explored further in the interaction energy decomposition section below.

Interaction Energy Decomposition. Although coupledcluster theory with single, double, and perturbative triple excitations (CCSD(T)), in conjunction with correlation-consistent basis sets (aug-cc-pVTZ and aug-cc-pVQZ) and a complete basis set extrapolation scheme, is capable of obtaining very accurate interaction energies, it does not help us understand how or why benzene-small molecule complexes bind the way they do. Therefore, we turn to the reduced variational space SCF method (RVS-SCF) to provide a decomposition of the total interaction energy into physically intuitive components. RVSSCF results for all benzene-small molecule complexes are presented in Table 6. From this table, we observe that electrostatic and dispersion interactions are the main stabilizing effects, while the main destabilizing influence is exchange repulsion. The relative contribution of electrostatics and dispersion forms a continuum, from predominantly dispersion-bound systems ( $\mathrm{Ne}$ and $\mathrm{Ar}$ ) through to systems with significant electrostatic interactions ( $\mathrm{HF}$ and $\mathrm{HCl}$ ). However, dispersion always plays a significant role in the binding of these systems,
TABLE 7: Est. CCSD(T)/aug-cc-pVQZ Optimized Intermolecular Distances $\left(R_{\mathrm{e}}\right)$ and $\operatorname{CCSD}(\mathrm{T}) / \mathrm{CBS}$ Torsional Barriers $\left(\Delta E=E_{\mathrm{e}}-E_{0}\right)^{a}$

\begin{tabular}{ccclll}
\hline molecule & $R_{\mathrm{e}}{ }^{\mathrm{cm}-\mathrm{X}}$ & $R_{\mathrm{e}}^{\mathrm{cm}-\mathrm{cm}}$ & \multicolumn{1}{c}{$R_{0}{ }^{\mathrm{cm}-\mathrm{cm}}$} & $\Delta E_{\text {twist }}$ & $\Delta E_{\text {swing }}$ \\
\hline $\mathrm{Ne}$ & 3.35 & 3.35 & $3.31-3.462$ & & \\
$\mathrm{HF}$ & 3.20 & 3.15 & 3.18 & & \\
$\mathrm{H}_{2} \mathrm{O}$ & 3.35 & 3.28 & $3.32-3.373$ & -0.0008 & -0.061 \\
$\mathrm{NH}_{3}$ & 3.60 & 3.58 & 3.59 & -0.325 & \\
$\mathrm{CH}_{4}$ & 3.80 & 3.80 & & -0.006 & \\
$\mathrm{Ar}$ & 3.55 & 3.55 & $3.52-3.59$ & & \\
$\mathrm{HCl}$ & 3.65 & 3.61 & 3.6291 & & \\
$\mathrm{H}_{2} \mathrm{~S}$ & 3.80 & 3.75 & 3.771 & 0.0007 & -0.076 \\
$\mathrm{PH}_{3}$ & 4.05 & 4.01 & & -0.143 & \\
$\mathrm{SiH}_{4}$ & 4.20 & 4.20 & & -0.034 &
\end{tabular}

${ }^{a}$ The $R_{\mathrm{e}}{ }^{\mathrm{cm}-\mathrm{X}}$ intermolecular distance is measured as the distance between the heteroatom of the molecule and the centre of the benzene ring, while $R_{\mathrm{e}}{ }^{\mathrm{cm}-\mathrm{cm}}$ denotes the center of mass to center of mass distance. $R_{0}{ }^{\mathrm{cm}-\mathrm{cm}}$ is the experimentally observed center of mass to center of mass. Where more than one value is available, a range covering all experimental data is given. All distances are reported in units of Ångstroms. All barrier heights are calculated at $\phi_{\text {twist }}=30$ or $\theta_{\text {swing }}=30$ and are reported in $\mathrm{kcal} / \mathrm{mol}$ relative to the energy of the starting structures $\left(\phi_{\text {twist }}=\theta_{\text {swing }}=0\right)$.

even when significant electrostatic interactions are also present. In fact, there are only three complexes, involving the strongly polar $\mathrm{HF}, \mathrm{HCl}$, and $\mathrm{H}_{2} \mathrm{O}$, where electrostatic interactions significantly outweigh dispersion interactions. Furthermore, the ratio of electrostatic stabilization to dispersion stabilization is lower for second-row halides than their first-row counterparts. This can be understood in terms of second-row halides having higher polarizabilities (i.e., more diffuse electron distributions) and, therefore, stronger dispersion interactions and also smaller dipole moments and, therefore, weaker electrostatic interactions.

The RVS-SCF results in Table 6 may also be compared with analogous SAPT2 results from the literature for the methane $\left(E_{\text {elst }}=-1.153, E_{\text {exch }}=2.164, E_{\text {disp }}=-2.025 \mathrm{kcal} / \mathrm{mol}\right)^{24}$ and hydrogen sulfide complexes $\left(E_{\text {elst }}=-3.18, E_{\text {exch }}=4.19, E_{\text {disp }}\right.$ $=-4.16 \mathrm{kcal} / \mathrm{mol}) .{ }^{10}$ Although the SAPT2 total and component energies differ from the RVS-SCF results by up to $21 \%$, the overall breakdown profile is similar, irrespective of the method used. This provides some assurance that both the SAPT2 and RVS-SCF methods are capturing the same physically based exchange, correlation, and electrostatic effects and suggests that either method may be applied to help explain the binding patterns of benzene-small molecule complexes.

Intermolecular Distances and Angles. Optimized intermolecular distances obtained from est. CCSD(T)/aug-cc-pVQZ PECs are presented alongside CCSD(T)/CBS rotational barrier heights in Table 7. For comparison purposes, experimental estimates of the intermolecular distances are also summarized in this table. Full details of all intermolecular interaction potentials, along with the equilibrium geometry of each complex (within the rigid monomer approximation), may be obtained as Supporting Information. From Table 7, we observe that equilibrium intermolecular distances generally decrease as the nuclear charge on the heteroatom increases, with the exception of neon, which lies further from the center of the benzene ring 
TABLE 8: Zero-Point Vibrational Energies (kcal/mol), Estimated As the Difference between the CCSD(T)/CBS Equilibrium Binding Energies $\left(D_{\mathrm{e}}\right)$ and the Set of All Experimentally Determined Vibrationally Averaged Binding Energies $\left(D_{0}\right)$

\begin{tabular}{cc}
\hline system & ZPVE \\
\hline $\mathrm{H}_{2} \mathrm{O}$ & $0.72-0.91$ \\
$\mathrm{NH}_{3}$ & $0.66-1.10$ \\
$\mathrm{CH}_{4}$ & $0.28-0.38$ \\
$\mathrm{Ar}$ & $0.15-0.25$ \\
$\mathrm{HCl}$ & 1.03
\end{tabular}

than the fluorine atom of hydrogen fluoride. This appears counterintuitive; steric arguments would suggest that neon should reside closer to the benzene ring than fluorine, the outermost atom in the benzene-HF complex. Upon further reflection, however, it is plausible that the electrostatic interaction between the highly polar HF molecule and the $\pi$ cloud of the benzene ring provides sufficient incentive for the $\mathrm{HF}$ molecule to overcome a significant degree of exchange repulsion and approach the benzene ring closer than would be expected from its van der Waals radius. Conversely, the relatively light and nonpolar neon atom has no such incentive and prefers to retreat from the benzene ring until its long-range dispersion interaction outweighs the short-range exchange repulsion. This hypothesis is confirmed by inspection of the RVS-SCF results presented in Table 6, which show that the strong electrostatic interaction between HF and benzene allows HF to overcome significant exchange repulsion and approach the benzene ring to well within its classical van der Waal's radius. The stark contrast between the strong electrostatic binding of HF and the very weak dispersion interaction between benzene and neon gives rise to the anomalous bond length behavior.

From Table 7, we note that all nonhalide molecules except $\mathrm{H}_{2} \mathrm{~S}$ prefer to adopt a staggered conformation relative to the benzene ring $\left(\phi_{\text {twist }}=30^{\circ}\right)$. However, previous work by Tauer et al. ${ }^{10}$ indicates that the barrier to $\mathrm{H}_{2} \mathrm{~S}$ twisting is so small that it can be considered negligible as zero-point motion allows the $\mathrm{H}_{2} \mathrm{~S}$ molecule to rotate freely about the $C_{2 v}$ axis of the symmetric starting structure $\left(\phi_{\text {twist }}=0^{\circ}\right)$. The present work confirms this result and indicates that the barrier to twisting motion is also negligible $(<0.05 \mathrm{kcal} / \mathrm{mol})$ for other systems including water, methane, and silane. Ammonia and phosphine both possess significant barriers to rotation of 0.33 and $0.14 \mathrm{kcal} / \mathrm{mol}$, respectively. Although these barriers are smaller than the estimated ZPVE of the ammonia-benzene complex $(0.66-1.10$ $\mathrm{kcal} / \mathrm{mol}$, Table 8), a large fraction of the ZPVE will be tied up in the intermolecular stretching mode; therefore, free rotation of these molecules above the plane of the benzene ring is unlikely. However, fully anharmonic calculations of the nuclear vibrational zero-point energy and wave function will be required to conclusively determine whether or not this is the case. The unusually high rotational barriers of $\mathrm{NH}_{3}$ and $\mathrm{PH}_{3}$ are most likely due to their lone pairs, which can behave differently according to the local environment, that is, whether the lone pair is above a bond or an atom of the benzene ring.

The results presented in Table 7 also show that benzene $-\mathrm{H}_{2} \mathrm{O}$ and benzene $-\mathrm{H}_{2} \mathrm{~S}$ complexes adopt equilibrium conformations with tilted molecules $\left(\theta_{\text {swing }}=30^{\circ}\right)$ sitting directly above the plane of the benzene ring. This results in a single proton pointing down toward the ring. However, it is known experimentally that the vibrationally averaged structures of both complexes have $C_{2 v}$ symmetry $\left(\theta_{\text {swing }}=0^{\circ}\right)$, which implies that their barriers to lateral swinging motion are lower than their zero-point vibrational energies. This is consistent with the theoretical finding that the ZPVE of the benzene $-\mathrm{H}_{2} \mathrm{O}$ complex $(0.63 \mathrm{kcal} / \mathrm{mol})^{16}$ is an order of magnitude larger than the barrier to swinging motion. Likewise, the ZPVE of the benzene $-\mathrm{H}_{2} \mathrm{~S}$ complex is expected to be approximately an order of magnitude larger than its barrier to swinging motion.

Comparison with Experiment. From Table 7, we observe that the calculated intermolecular distances differ by at most $0.04 \AA$ from the nearest experimental estimate for all systems where experimental results are available $\left(\mathrm{Ne}, \mathrm{HF}, \mathrm{H}_{2} \mathrm{O}, \mathrm{NH}_{3}\right.$, $\left.\mathrm{Ar}, \mathrm{HCl}, \mathrm{H}_{2} \mathrm{~S}\right)$. It is encouraging that these deviations lie within the resolution to which intermolecular distances have been determined here $(0.05 \AA)$. However, it is worth keeping in mind that the experimentally measured bond length implicitly includes the effect of zero-point vibrational motion, which has not yet been taken into account. Previous work by Gregory and Clary ${ }^{16}$ has shown that the vibrationally averaged distance between the centers of mass of benzene and water is around $0.1 \AA$ greater than their equilibrium separation. This is consistent with the fact that predicted equilibrium distances are almost always lower than their experimentally determined vibrationally averaged counterparts. The equilibrium distances reported here should be considered as a lower bound to the experimentally observable vibrationally averaged distances.

An extensive survey of previous experimental and high-level theoretical determinations of the binding energy is provided in Table 1. Equilibrium binding energies $\left(D_{\mathrm{e}}\right)$ derived from the data presented in Tables 3 and 7 are also presented in Table 1. To enable direct comparison between theory and experiment, it is necessary to take into account the zero-point vibrational energy of each complex. However, the only complex for which a reliable theoretical value of the ZPVE is currently available is benzene $-\mathrm{H}_{2} \mathrm{O}$, for which the quantum diffusion Monte Carlo simulations of Gregory and Clary yield a value of $0.63 \mathrm{kcal} /$ mol. ${ }^{16}$ Adding this ZPVE to the equilibrium binding energy of $-3.16 \mathrm{kcal} / \mathrm{mol}$ yields a vibrationally averaged binding energy of $-2.53 \mathrm{kcal} / \mathrm{mol}$ for this system. This is in good agreement with the most recent experimental value of $-2.44 \mathrm{kcal} / \mathrm{mol}^{31}$ For the other complexes, ZPVEs can be estimated by taking the difference between experimental $\left(D_{0}\right)$ and theoretical $\left(D_{\mathrm{e}}\right)$ binding energies, yielding the ZPVE ranges presented in Table 8. However, for genuine de novo prediction of vibrationally averaged binding energies, it is necessary to calculate ab initio zero-point vibrational energies for each complex. This requires the construction of accurate ab initio potential energy surfaces and fully anharmonic calculation of the zero-point vibrational energy. The potential energy curves calculated in this work provide a useful starting point for this process.

\section{Conclusions}

Binding energies and potential energy curves for the interaction between benzene and a series of first- and second-row hydrides and rare gas atoms were calculated at est. $\operatorname{CCSD}(\mathrm{T}) /$ aug-cc-pVQZ. Application of a complete basis set extrapolation procedure showed that these results are very close to the complete basis set limit, with basis set incompleteness errors of less than $0.1 \mathrm{kcal} / \mathrm{mol}$ in all cases. Likewise, the error arising from neglect of post-triples contributions to the correlation energy was estimated to be less than $0.07 \mathrm{kcal} / \mathrm{mol}$. Equilibrium intermonomer distances and orientations were found to be entirely consistent with previous theoretical and experimental results. Altogether, these results provide suitable benchmark data for the development and testing of more approximate and more efficient post-Hartree-Fock methods for calculating long-range and noncovalent interaction energies. However, to enable direct 
comparison with experiment, the effects of zero-point nuclear vibrational motion must be accounted for. The potential energy curves obtained in this work represent a first step toward acquiring the high-quality potential energy surfaces needed for accurate determination of the anharmonic zero-point energy and vibrationally averaged structures and properties.

Acknowledgment. The author would like to thank the Australian National Computing Infrastructure Scheme and the Australian National University Supercomputer Facility for generous grants of computer time and the ARC for funding (DP0771978).

Supporting Information Available: The equilibrium geometries of all complexes and est. CCSD(T)/aug-cc-pVQZ potential energy curves. This material is available free of charge via the Internet at http://pubs.acs.org.

\section{References and Notes}

(1) Adcock, S. A.; McCammon, J. A. Chem. Rev. 2006, 106, 1589.

(2) Grimme, S. J. Comput. Chem. 2004, 25, 1463.

(3) Becke, A. D.; Johnson, E. R. J. Chem. Phys. 2005, 122, 154104.

(4) Johnson, E. R.; Becke, A. D. J. Chem. Phys. 2005, 123, 024101.

(5) Becke, A. D.; Johnson, E. R. J. Chem. Phys. 2005, 123, 154101.

(6) Becke, A. D.; Johnson, E. R. J. Chem. Phys. 2006, 124, 014104.

(7) Johnson, E. R.; Becke, A. D. J. Chem. Phys. 2006, 124, 174104

(8) Johnson, E. R.; Becke, A. D. Chem. Phys. Lett. 2006, 432, 600

(9) Becke, A. D.; Johnson, E. R. J. Chem. Phys. 2007, 127, 154108.

(10) Tauer, T. P.; Derrick, M. E.; Sherrill, C. D. J. Phys. Chem. A 2005 109, 191.

(11) Hobza, P.; Bludskg, O.; Selzle, H. L.; Schlag, E. W. J. Chem. Phys. 1992, 97, 335

(12) Klopper, W.; Lüthi, H. P.; Brupbacher, Th.; Bauder, A. J. Chem. Phys. 1994, 101, 9747.

(13) Rozas, I.; Alkorta, I.; Elguero, J. J. Phys. Chem. A 1997, 101, 9457.

(14) Mishra, B. K.; Sathyamurthy, N. J. Phys. Chem. A 2007, 111, 2139

(15) Tarakeshwar, P.; Choi, H. S.; Kim, K. S. J. Am. Chem. Soc. 2001, 123,3323 .

(16) Gregory, J. K.; Clary, D. C. Mol. Phys. 1996, 88, 33.

(17) Feller, D. J. Phys. Chem A 1999, 103, 7558.

(18) Tsuzuki, S.; Honda, K.; Uchimaru, T.; Mikami, M.; Tanabe, K. J. Am. Chem. Soc. 2000, 122, 11450.

(19) Min, S. K.; Lee, E. C.; Lee, H. M.; Kim, D. Y.; Kim, D.; Kim, K. S. J. Comput. Chem. 2008, 29, 1208.

(20) Jurecka, P.; Sponer, J.; Cerny, J.; Hobza, P. Phys. Chem. Chem. Phys. 2006, 8, 1985.

(21) Hobza, P.; Havlas, Z. Chem. Rev. 2000, 100, 4253.

(22) Tsuzuki, S.; Honda, K.; Uchimaru, T.; Mikami, M.; Tanabe, K. J. Am. Chem. Soc. 2000, 122, 3746.

(23) Morita, S.; Fujii, A.; Mikami, M.; Tsuzuki, S. J. Phys. Chem. A 2006, 110, 10583.

(24) Ringer, A. L.; Figgs, M. S.; Sinnokrot, M. O.; Sherrill, C. D. J. Phys. Chem. A 2006, 110, 10822 . 198.

(25) Koch, H.; Fernandez, B.; Makarewicz, J. J. Chem. Phys. 1999, 111,

(26) Tarakeshwar, P.; Lee, S. J.; Lee, J. Y.; Kim, K. S. J. Chem. Phys. 1998, 108, 7217.

(27) Cappelletti, D.; Bartolomei, M.; Pirani, F.; Aquilanti, V. J. Phys. Chem. A 2002, 106, 10764.

(28) Brupbacher, Th.; Makarewicz, J.; Bauder, A. J. Chem. Phys. 1994, 101,9736 .

(29) Baiocchi, F. A.; Williams, J. H.; Klemperer, W. J. Phys. Chem. 1983, 87, 2079.

(30) Cheng, B.-M.; Grover, J. R.; Walters, E. A. Chem. Phys. Lett. 1995, 232,364 .

(31) Courty, A.; Mons, M.; Dimicoli, I.; Piuzzi, F.; Gaigeot, M.-P.; Brenner, V.; de Pujo, P.; Millie, P. J. Phys. Chem. A 1998, 102, 6590.
(32) Gutowsky, H. S.; Emilsson, T.; Arunan, E. J. Chem. Phys. 1993, $99,4883-33$

(33) Suzuki, S.; Green, P. G.; Bumgarner, R. E.; Dasgupta, S.; Goddard, W. A.; Blake, G. A. Science 1992, 257, 942.

(34) Gotch, A. J.; Zwier, T. S. J. Chem. Phys. 1992, 96, 3388.

(35) Mons, M.; Dimicoli, I.; Tardivel, B.; Piuzzi, F.; Brenner, V.; Millie, P. Phys. Chem. Chem. Phys. 2002, 4, 571.

(36) Rodham, D. A.; Suzuki, S.; Suenram, R. D.; Lovas, F. J.; Dasgupta, S.; Goddard, W. A.; Blake, G. A. Nature 1993, 362, 735.

(37) Shibasaki, K.; Fujii, A.; Mikami, N.; Tsuzuki, S. J. Phys. Chem. A 2006, 110, 4397

(38) Krause, H.; Neusser, H. J. J. Chem. Phys. 1993, 99, 6278.

(39) Weber, Th.; von Bargen, A.; Riedle, E.; Neusser, H. J. J. Chem. Phys. 1990, 92, 90.

(40) Satink, R. G.; Piest, H.; von Helden, G.; Meijer, G. J. Chem. Phys. 1999, 111, 10750.

(41) Read, W. G.; Campbell, E. J.; Henderson, G. J. Chem. Phys. 1983, 78,3501 .

(42) Gotch, A. J.; Zwier, T. S. J. Chem. Phys. 1990, 93, 6977.

(43) Walters, E. A.; Grover, J. R.; White, M. G.; Hui, E. T. J. Phys. Chem. 1985, 89, 3814.

(44) Mons, M.; Dimicoli, I.; Piuzzi, F. Int. Rev. Phys. Chem. 2002, 21, 101.

(45) Arunan, E.; Emilsson, T.; Gutowsky, H. S.; Fraser, G. T.; de Oliveira, G.; Dykstra, C. E. J. Chem. Phys. 2002, 117, 9766.

(46) Gauss, J.; Stanton, J. F. J. Phys. Chem. A. 2000, 104, 2865.

(47) Müller, H.; Franke, R.; Vogtner, S.; Jaquet, R.; Kutzelnigg, W. Theor. Chem. Acc. 1998, 100, 85.

(48) Polyansky, O. L.; Csaszar, A G.; Shirin, S. V.; Zobov, N. F.; Barletta, P.; Tennyson, J.; Schwenke, D. W.; Knowles, P. J. J. Am. Chem. Soc. 2003, 299, 539 .

(49) Demaison, J.; Margules, L.; Boggs, J. E. Chem. Phys. 2000, 260, 65 .

(50) Stanton, J. F. Mol. Phys. 1999, 97, 841.

(51) Styszynski, J. Chem. Phys. Lett. 2000, 317, 351.

(52) Edwards, T. H.; Moncur, N. K.; Snyder, L. E. J. Chem. Phys. 1967, 46, 2139.

(53) Wang, D.; Shi, Q.; Zhu, Q.-S. J. Chem. Phys. 2000, 112, 9624. 945 .

(55) Raghavachari, K.; Trucks, G. W.; Pople, J. A.; Head-Gordon, M. Chem. Phys. Lett. 1989, 157, 479.

(56) Dunning, T. H., Jr. J. Chem. Phys. 1989, 90, 1007.

(57) Woon, D. E.; Dunning, T. H., Jr. J. Chem. Phys. 1993, 98, 1358.

(58) Boys, S. F.; Bernardi, F. Mol. Phys. 1970, 19, 553.

(59) van Duijneveldt, F. B.; van Duijneveldt-van de Rijdt, J. G. C. M.; van Lenthe, J. H. Chem. Rev. 1994, 94, 1873.

(60) Helgaker, T.; Klopper, W.; Koch, H.; Noga, J. J. Chem. Phys. 1997, 106,9639 .

(61) Chen, W.; Gordon, M. S. J. Phys. Chem. 1996, 100, 14316.

(62) Schmidt, M. W.; Baldridge, K. K.; Boatz, J. A.; Elbert, S. T.; Gordon, M. S.; Jensen, J. H.; Koseki, S.; Matsunaga, N.; Nguyen, K. A.; Su, S. J.; Windus, T. L.; Dupuis, M.; Montgomery, J. A. J. Comput. Chem. $1993,14,1347$.

(63) Werner, H.-J.; Knowles, P. J.; Lindh, R.; Manby, F. R.; Schütz, M.; Celani, P.; Korona, T.; Rauhut, G.; Amos, R. D.; Bernhardsson, A.; Berning, A.; Cooper, D. L.; Deegan, M. J. O.; Dobbyn, A. J.; Eckert, F.; Hampel, C.; Hetzer, G.; Lloyd, A. W.; McNicholas, S. J.; Meyer, W.; Mura, M. E.; Nicklass, A.; Palmieri, P.; Pitzer, R.; Schumann, U.; Stoll, H.; Stone, A. J.; Tarroni, R.; Thorsteinsson, T. MOLPRO, version 2006.1, a package of ab initio programs; see: http://www.molpro.net.

(64) Grimme, S. J. Chem. Phys. 2003, 118, 9095.

(65) Bachorz, R. A.; Bischoff, F. A.; Höfener, S.; Klopper, W.; Ottiger, P.; Leist, R.; Frey, J. A.; Leutwyler, S. Phys. Chem. Chem. Phys. 2008, 10, 2758.

(66) Takatani, T.; Sherrill, C. D. Phys. Chem. Chem. Phys. 2007, 9 , 6106.

(67) Hopkins, B. W.; Tschumper, G. S. J. Phys. Chem. A 2004, 108, 2941.

(68) Pittner, J.; Hobza, P. Chem. Phys. Lett. 2004, 390, 496.

JP809106B 